\title{
Tech transfer revs up
}

Stacy Lawrence

New company formation (about half of which is biotech) from US research institutions was way up in 2004 (up to >460). In 2003, biotech startup growth in US and Europe slowed, with Denmark and Ireland 고 showing highest relative rates of venture formation. In FY2004, US patent

\section{Tech transfer staffing at US universities}

More universities are dedicating staff to tech transfer and existing staff size is increasing.

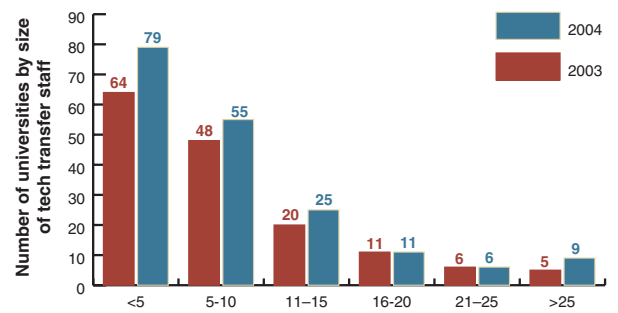

Source: Association of University Technology Managers

\section{Change in US university tech transfer indicators}

Research expenditures were all down in 2004, but aggregate patent applications are up

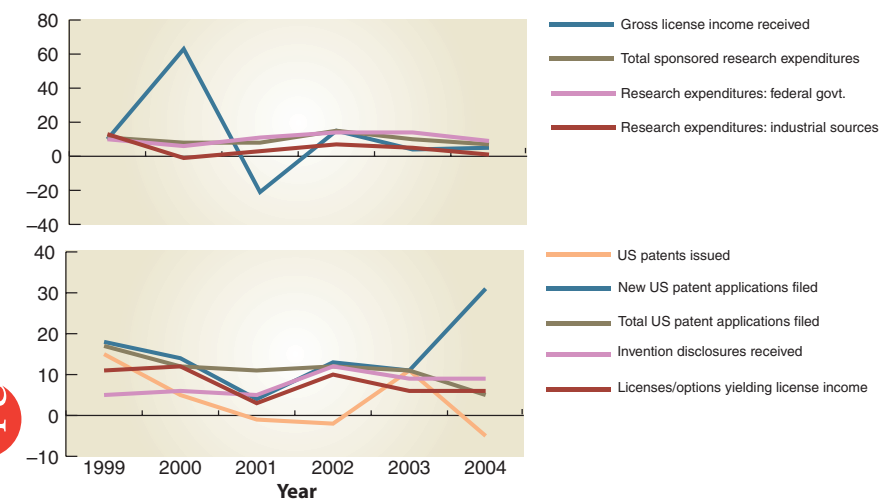

Source: Association of University Technology Managers

Tech transfer at top US research institutions in 2004

\begin{tabular}{|c|c|}
\hline Institution name & $\begin{array}{c}\text { Research } \quad \text { Lic } \\
(\$ \text { millions })^{a} \quad(\$ \mathrm{mi}\end{array}$ \\
\hline \multicolumn{2}{|l|}{ Universities } \\
\hline Univ. of California System ${ }^{i}$ & 2792 \\
\hline Johns Hopkins Univ. ${ }^{i}$ & 1595 \\
\hline Massachusetts Inst. of Technology (MIT) & 1027 \\
\hline Univ. of Washington/Wash. Research Fdn. ${ }^{i}$ & 834 \\
\hline Univ. of Illinois, Chicago, Urbana ${ }^{i}$ & 814 \\
\hline Univ. of Wisconsin at Madisoni & 764 \\
\hline Univ. of Michigan ${ }^{i}$ & 753 \\
\hline SUNY Research Fdn. ${ }^{i}$ & 710 \\
\hline Stanford Univ.i & 694 \\
\hline Univ. of Pennsylvaniai & 654 \\
\hline
\end{tabular}

Hospitals and research institutes

The General Hospital dba Massachusetts General Hospital -463

Mayo Fdn. for Medical Education and Research 372

Brigham \& Women's Hospital

M.D. Anderson Cancer Ctr.

Sloan Kettering Inst. for Cancer Res.

Fred Hutchinson Cancer Res. Ctr.

Beth Israel Deaconess Medical Ctr.

Dana-Farber Cancer Inst.

Children's Hospital, Cincinnati

St. Jude Children's Research Hospital

$\begin{array}{lcc}463 & 63 & 245 \\ 372 & 22 & 310 \\ 363 & 8 & 104 \\ 314 & 5 & 115 \\ 239 & 76 & 47 \\ 222 & 3 & 14 \\ 182 & 3 & 72 \\ 170 & 4 & 66 \\ 150 & 3 & 32 \\ 142 & 1 & 35\end{array}$

incol

\section{New biotech company formation in Europe/US}

New company formation in 2003 continued to be slow, although startup activity in Ireland was buoyant

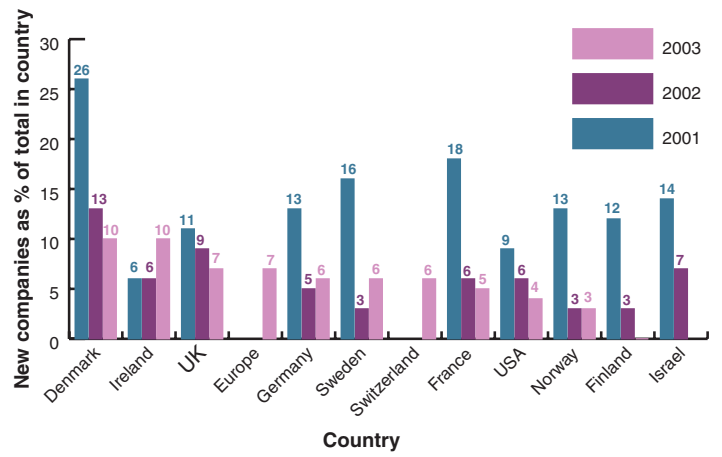

Source: Critical

\begin{tabular}{|c|c|c|c|c|c|c|}
\hline $\begin{array}{l}\text { Patents } \\
\text { filed }^{\mathrm{d}}\end{array}$ & $\begin{array}{l}\text { Executed } \\
\text { licenses }^{\mathrm{e}}\end{array}$ & $\begin{array}{l}\text { Paying } \\
\text { licenses }\end{array}$ & $\begin{array}{c}>\$ 1 \text { million } \\
\text { licenses }\end{array}$ & $\begin{array}{l}\text { Patents } \\
\text { issued }\end{array}$ & $\begin{array}{l}\text { Companies } \\
\text { formed }\end{array}$ & $\begin{array}{l}\text { Companies } \\
\text { operational }\end{array}$ \\
\hline 965 & 273 & 906 & 15 & 270 & 5 & 206 \\
\hline 586 & 100 & 197 & 0 & 89 & 5 & 45 \\
\hline 436 & 134 & 410 & 4 & 159 & 20 & 157 \\
\hline 133 & 70 & 322 & 5 & 38 & 7 & $\mathrm{~N} / \mathrm{A}$ \\
\hline 196 & 88 & 164 & 1 & 59 & 16 & 52 \\
\hline 217 & 203 & 261 & 8 & 93 & 2 & 30 \\
\hline 204 & 73 & 172 & 3 & 74 & 13 & 76 \\
\hline 172 & 50 & 157 & 1 & 43 & 7 & 43 \\
\hline 277 & 89 & 474 & 6 & 87 & 9 & 99 \\
\hline 287 & 87 & 54 & 1 & 45 & 6 & 43 \\
\hline 288 & 75 & 144 & 5 & 73 & 3 & 39 \\
\hline 147 & 97 & 268 & 3 & 28 & 3 & 11 \\
\hline 98 & 40 & 76 & 1 & 37 & 5 & 17 \\
\hline 46 & 33 & 41 & 1 & 19 & 2 & 11 \\
\hline 75 & 28 & 70 & 4 & 22 & 4 & 9 \\
\hline 17 & 11 & 72 & 0 & 2 & 0 & 8 \\
\hline 84 & 20 & 51 & 0 & 21 & 1 & 17 \\
\hline 61 & 34 & 135 & 1 & 15 & 2 & 7 \\
\hline 51 & 17 & 23 & 1 & 6 & 3 & 3 \\
\hline 24 & 28 & 106 & 0 & 5 & 0 & 0 \\
\hline
\end{tabular}

